\title{
Increasing Pilot's Understanding of Future Automation State - An Evaluation of an Automation State and Trajectory Prediction System
}

\author{
Timothy J. Etherington \\ Technical Fellow \\ Collins Aerospace \\ Hampton, Virginia \\ timothy.j.etherington@nasa.gov
}

\author{
Lynda J. Kramer \\ Crew Systems and Aviation \\ Operations Branch \\ NASA Langley Research Center \\ Hampton, Virginia \\ lynda.j.kramer@nasa.gov
}

Emory T. Evans

Flight Software Systems Branch

NASA Langley Research Center

Hampton, Virginia

e.t.evans@nasa.gov

\author{
Kimberlee H. Shish \\ Intelligent Systems Division \\ NASA Ames Research Center \\ Moffett Field, CA \\ kimberlee.h.shish@nasa.gov
}

Taumi S. Daniels

Electronic Instrument Systems Branch

NASA Langley Research Center

Hampton, Virginia

taumi.s.daniels@nasa.gov

\author{
Steven D. Young \\ Safety-Critical Avionics Systems \\ Branch \\ NASA Langley Research Center \\ Hampton, Virginia \\ steven.d.young@nasa.gov
}

\footnotetext{
conducted at NASA Langley Research Center to evaluate a trajectory prediction system. The trajectory prediction system computes a five-minute prediction of the lateral and vertical path of the aircraft given the current and intent state of the automation. The prediction is shown as a graphical representation so the pilots can form an accurate mental model of the future state. Otherwise, many automation changes and triggers are hidden from the flight crew or need to be consolidated to understand if a change will occur and the exact timing of the change. Varying dynamic conditions like deceleration can obscure the future trajectory and the ability to meet constraints, especially in the vertical dimension. Current flight deck indications of flight path assume constant conditions and do not adequately support the flight crew to make correct judgments regarding constraints.

The study was conducted using ten commercial airline crews from multiple airlines, paired by airline to minimize procedural effects. Scenarios spanned a range of conditions that provided evaluation in a realistic environment with complex traffic and weather conditions. In particular, scenarios probed automation state and loss of state awareness. The technology was evaluated and contrasted with current state-of-the-art flight deck capabilities modeled from the Boeing 787.

Objective and subjective data were collected from aircraft parameters, questionnaires, audio/video recordings, head/eye tracking data, and observations. This paper details findings about the trajectory prediction system including recommendations about further study.

Keywords-flight crew error, aviation safety, synoptic displays, automation, automation surprise, unreliable airspeed, hydraulic systems failure
}

\section{INTRODUCTION}

Automation complexity and non-transparency continue to create situations where pilots are surprised during routine operations [1]. Navigation and flight management systems in particular create optimized routing but only the planned route (i.e., flight plan) is shown to the pilot. If conditions are encountered outside the plan or if automation modes do not allow the execution of the plan, pilots are sometimes surprised by the automation, often expressing "what is it doing now" or "what is it doing next" [2]. In addition, air traffic control (ATC) constraints rarely allow for completion of the plan as originally constructed. Once off the plan, current tools like instantaneous flight path vector or range to target altitude are only useful during stabilized flight conditions; they do not allow preview during changing flight conditions. Without preview, pilots tend to aggressively manage energy with speed brakes during idle descent.

A trajectory prediction system (TPS) is proposed to provide a preview into the operation of the automation [3]. In addition to the flight management system (FMS) plan described by the magenta line on the displays, a green line is provided for some time into the future that represents what the aircraft will actually fly given the current setup of the autoflight system. This trajectory prediction takes into account actual conditions and the automation state. It includes all the rules that are hidden from the pilot in the complex interaction between the flight management system, the autopilot, and any manual constraints that are imposed from the mode control panel. This preview may be used, for example, to accurately predict if altitude constraints will be met and if/when the aircraft will get back on path without pilot intervention (such as deploying drag devices).

A simulation experiment was conducted to investigate the utility and pilot acceptability of such a TPS in response to 
aircraft awareness research objectives [4, 5, 6, 7]. The study was conducted using ten commercial airline crews from multiple airlines, paired by airline to minimize procedural effects. Scenarios spanned a range of conditions that provided evaluation in a realistic environment with complex traffic and weather conditions. This paper adds to prior work [3][8], providing findings about the TPS using a high-fidelity simulation environment and platform involving complex scenarios.

\section{BACKGROUND}

Vertical navigation (VNAV) planning before the introduction of onboard computer-based systems consisted mainly of climbout at specified power settings and nominal vertical speed rates to the cruise altitude, fuel burn at the planned cruise altitude and distance to destination, and a planned vertical speed descent to the destination airport. For the descent phase, pilots developed easy to remember rules of thumb for the distance to start from the destination to initiate the descent and a rate for required vertical speed. The "rule of three" is three miles for every 1000 feet of altitude to lose. The "rule of five" is to achieve a three degree descent multiply your ground speed by five to get the required vertical speed.

The first implementation of a VNAV FMS [9] was in 1982 on the B-757/767 aircraft. Original implementation was for enroute optimization with no RNAV or RNP capability and limited descent capability. The FMS VNAV was enhanced and improved as performance-based operations matured including the use of a cost index to balance fuel against hourly related costs. VNAV provided guidance for autopilot, flight director and autothrottle. VNAV provided speed-based climb and cruise economics. Then climb, based on economy or a pilot-selected speed, was implemented. Performance was based on aircraft type, gross weight, anti-ice, winds, and temperature.

Later, VNAV performance planning for descents was implemented providing reduced workload on complex arrivals. The FMS would meet all speed and altitude restrictions while honoring airplane operating limits. The VNAV path can be constructed as a performance path or a geometric path. The performance path is planned as an idle descent from top of descent to the first constrained waypoint. Geometric path can be a point-to-point between two constrained waypoints or a flight path angle. These are typically shallower and not an idle path descent.

Computer-based VNAV at its introduction was a powerful tool for managing flight in a constrained environment but navigation displays typically only provide a lateral $2 \mathrm{D}$ presentation [10, 11]. The FMS only provided vertical speed targets and vertical deviation indications to pilots to judge if the system was following the planned profile. The vertical presentation of the plan was missing and resulted in missed constraints and mistrust of the automation. Pilots had to visualize which segment of the arrival they were currently on and what speed and altitude constraints were required. In addition, they needed to judge whether future constraints could be met as well. There was no pictorial view in the cockpit for the vertical profile. In 2003, a custom option was designed for a vertical situation display (VSD) on the B-737 for forward and retro-fit that provided a slice of the vertical path below the lateral

NASA's Airspace Operations and Safety Program, Safe Autonomous Systems Operations Project. path on the navigation display moving map. The VSD [12] is now an option on the B-777 and B-787 as well as the Airbus A350.

With the aircraft ability to fly complex arrivals, standard arrival procedures have proliferated to improve airspace operating efficiencies and controller workload, meet conflicting airspace constraints, impose noise abatement procedures, or combinations of each. Many of the arrivals also end in an RNAV/RNP procedure that may be curved and complex with additional speed and altitude constraints. Often, ATC changes the plan due to traffic conditions resulting in aircrews too frequently missing some of the route constraints. The VSD shows the prior plan and the instantaneous vertical speed of the aircraft but nothing about the predicted future state. Especially when changing conditions due to a speed restriction, the current vertical speed provides little information about the future state when the new speed is attained. The VSD does not show upcoming automation changes to other modes, even if the system knows the mode will change. To manage changing constraints that are near the performance capability of the aircraft, pilots will often aggressively manage energy utilizing drag devices at the cost of performance and fuel.

A TPS was developed at NASA Ames Research Center [3, 8] to address this deficiency. Information is added to the existing VSD that utilizes information from the FMS intent bus in order to predict more closely the vertical path the automation will actually attain and show upcoming automation state changes as well. The system utilizes current atmospheric conditions and will show deviation due to wind. Since predictors become less accurate due to forecast uncertainties, the TPS shows the predicted flight path out five minutes.

\section{METHOD}

A pilot-in-the-loop flight simulation study was conducted at NASA Langley Research Center to evaluate the TPS aimed at supporting the flight crew's awareness of problems related to energy states. The TPS indicators were evaluated and compared utilizing state-of-the-art flight deck systems common on modern commercial air transport aircraft.

The pilot-in-the-loop study was conducted using commercial airline crews in a full-mission high-fidelity flight simulator. Crews were paired by airline for common crew resource management procedures and protocols. Scenarios spanned a range of complex conditions while emulating several causal factors reported in recent accidents involving loss of energy state awareness by pilots. Ten U.S. airline crews flew various normal and non-normal procedures and their actions and performance were recorded in response to these conditions.

Data were collected via questionnaires administered at the completion of flight scenarios, aircraft parameters, audio/video recordings, eye tracking, pilot control inputs, and researcher observations. Questionnaire response data included subjective measures of workload, situation awareness, complexity, decision making, state awareness, usability, and acceptability. This paper reports relevant findings derived from subjective measures for the TPS only. 


\section{A. Experiment Design}

The experiment used a between-subjects design to evaluate a number of technologies during nominal and off-nominal flight conditions. Each crew flew eight runs, two nominal training and familiarization flights, one from takeoff to landing and one from top of descent, and six off nominal flights, one from takeoff to failure and five from top of descent to landing. A nominal flight from takeoff to landing was used as a baseline run for workload, crew resource management, and other subjective measures. Offnominal runs consisted of relevant systems failures and autothrottle malfunctions. There were no specific off-nominal conditions for the TPS and the display technology was evaluated using normal ATC speed and altitude interventions from standard arrivals.

Two display configurations were compared using pilot comments and observations - a "baseline" with B-787-like displays [13] and a TPS that provided additional information on the navigation display (ND) and VSD.

\section{B. Trajectory Prediction System}

The TPS was developed at NASA Ames Research Center. Implementation and algorithmic details are found in the initial testing previously reported [3][8]. In simple terms, TPS provides a five minute prediction of the path of the aircraft and indicates when automation mode changes will occur and what the effect of those changes will be on the flight path. Aircraft performance and environmental conditions are considered in making the prediction. The system assumes the current configuration (current gear and flaps settings, no new automation inputs) for the duration of the prediction (no prior schedule assumed in advance), but it responds (i.e., the prediction updates) immediately to pilot interventions, such as deploying flaps, gear, and other drag devices. The predicted path is differentiated from the planned path (magenta line) by making the predicted path line green and displaying that prediction on both the lateral part of the ND and on the VSD when it is displayed below the lateral part of the ND, see Fig. 1.

A green circle symbol and label indicates where a mode change will occur and what the new mode will be. Pilot inputs cause the predictions to update and the indicators to change based on that input (e.g., selected speed changes, deploying speed brakes, or changing the altitude pre-selector). Mode changes that may be indicated include LNAV, LOC, ALT CAP, ALT HLD, VNAV PTH, VNAV SPD, G/S, and Flare. Indicated modes are aircraft type specific. Flap and speed changes commanded by the FMS are indicated by a green circle and label as well but are not part of TPS.

Fig. 1 provides an example of TPS indicators following a typical intervention by ATC such as was used in the experiment scenarios. The magenta line shown on the ND and VSD was planned using the FMS before top of descent. At a cost index of 100 as the fuel versus time number, the FMS plans for an idle descent around 290 knots to the first constrained waypoint at CAMRN which is to cross CAMRN at 250 knots and 11,000 feet. At top of descent, ATC clears the aircraft to descend but requests the aircraft to slow to 250 knots early due to traffic flow. This is reflected in a predicted flight path (green line) that deviates from the magenta path line. Modern jet aircraft have a difficult time slowing down while descending at the same time.
Aircraft will typically shallow the descent while transitioning to a slower airspeed. Careful study of Fig. 1 shows the magenta path (i.e., the planned path) on an idle descent at 290 knots and a deceleration profile (shallower angle descent while slowing down) before CAMRN to slow to 250 knots for the arrival restriction of CAMRN at 11,000 feet and at 250 knots. Picture a hockey stick as representing this plan. When ATC slows the

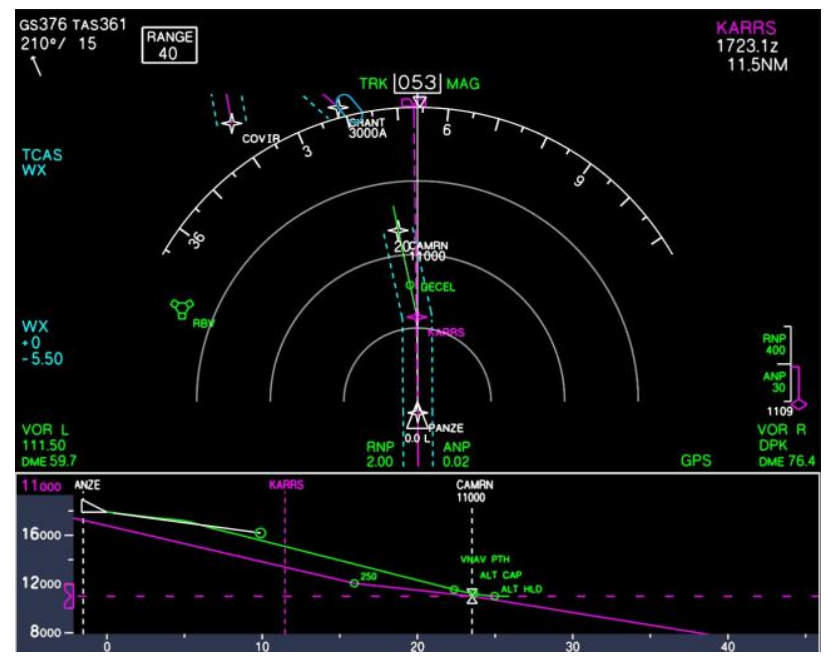

Fig. 1. TPS Indicators on ND and VSD

aircraft early, this plan becomes essentially inverted. The deceleration profile is conducted first followed by the idle descent phase. This initially puts the aircraft above the planned path and it appears that it will no longer meet the altitude constraint at CAMRN but since the airspeed is slower, the idle descent rate at 250 knots is greater than the descent rate at 290 knots. If you flip the hockey stick over you will see the paths are almost mirror images and the result is that the green predicted path and the magenta planned path will merge together at the constrained waypoint without further pilot intervention. If you mentally now remove the green line you will be left with a pictorial of the aircraft well above the path and the white line extending in front of the aircraft symbol on the VSD representing the instantaneous flight path angle indicating that we will not descend in time to meet the waypoint constraint. This conflicting (and potentially misleading) information occurs because the aircraft is currently decelerating and will not remain in this state for very long. In other words, without prediction the picture is not complete. When presented with the conflicting information, pilots almost always will deploy speed brakes and force the aircraft back onto the planned path, wasting energy and fuel and potentially subjecting the passengers to noise and objectionable movements.

\section{Apparatus}

The experiment was conducted in the NASA Langley Research Center (LaRC) Research Flight Deck (RFD) within the Cockpit Motion Facility (CMF), Fig. 2. Although initially designed as a B-757 flight deck, the RFD has been modified to emulate most of the B-787 displays and functions. This was done to utilize B-787-like capabilities as the reference flight deck when considering new technology concepts under the research objectives. 


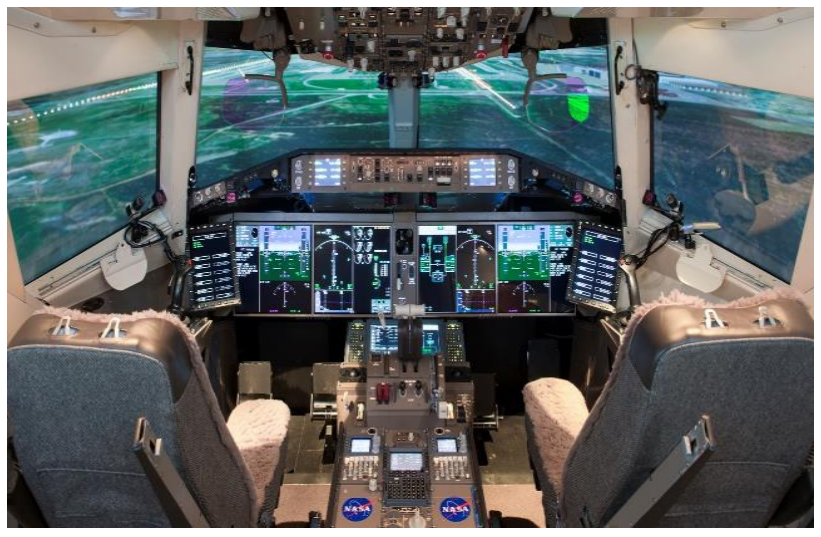

Fig. 2. Integration Flight Deck Simulator

The study was conducted in full motion with the RFD articulated on top of a hexapod hydraulic motion system. This facility incorporates fully functioning pilot controls, FMS, and six representative flight displays (2 PFDs, 2 MFDs, EICAS display and lower system display). EFB displays are installed outboard on each pilot's side and were used for charts and procedures (i.e., checklists). A collimated out-the-window scene is produced by a Collins Aerospace graphics system providing approximately 200 degree horizontal by 40 degree vertical fieldof-view at 26 pixels per degree. Head-up displays were stowed and not used for this study.

\section{Participants}

Twenty pilots (10 crews), representing three airlines, participated in this experiment. Each pilot held an Airline Transport Pilot rating and was current in the wide-body Boeing aircraft as either Captain or First Officer. Crews were paired from the same airline to minimize inter-crew conflicts in Standard Operating Procedures (SOPs) and Crew Resource Management (CRM) training. 17 participants were male and 3 were female. The Captains' average age was 60 years with an average of 25,400 total flight hours. The First Officers' average age was 55 years with an average of 14,831 total flight hours.

Pilot flying $(\mathrm{PF})$ and pilot monitoring (PM) roles were assigned based on the experimental matrix.

\section{E. Training}

Pilots were type-rated and qualified in wide-body Boeing aircraft. The simulator was a representation of that class of aircraft, including a mix of legacy B-757 systems with B-787 displays and controls and a software FMS representative of the B-737. Flight controls consisted of a side stick controller, rather than the control wheel and column device. Datalink messages were utilized for the majority of ATC interactions. Each of the items not on the aircraft type that pilots were current in, were briefed and reviewed in the simulator, and formal tests did not begin until the pilots said they were comfortable to proceed.

An extensive briefing about new technologies being evaluated was provided, followed by a training session on how to use and evaluate the new technologies with both pilots seated in the simulator. A familiarization run from takeoff to landing provided further training opportunities and allowed for training opportunities in normal dynamic conditions. TPS was explained and a video was shown using the standard arrival procedure with the ATC speed intervention that was utilized in the experiment. This same arrival and speed intervention was demonstrated in the nominal run.

\section{F. Procedures}

The route of flight flown by the pilots consisted of the DOCTR3 departure from the Ronald Reagan Washington National Airport (KDCA), proceeding enroute at FL190, then flying the CAMRN4 arrival into the John $F$ Kennedy International Airport (KJFK), which connected to special RNP RNAV procedure for Runway $13 \mathrm{~L}$ at JFK. The route duration was approximately 45 minutes. Changing weather conditions required ATC to route the aircraft to Runways 13R and 04R for some scenarios.

The entire flight was flown as a baseline run from takeoff to landing to provide a basis and context for the experiment. The majority of the non-normal runs were then initialized from just outside the top of descent point demonstrated in the nominal run.

Material representing some of the information provided by flight dispatch was given to the pilots, to include a detailed flight plan, weather forecasts, and applicable Notices to Airmen.

Normal aircraft traffic was modeled for a portion of the eastern seaboard of the United States and the New York area, including traffic in and out of LaGuardia and JFK. Scripted and computer-generated ATC voice communications was provided for all modeled aircraft and frequencies, including normal communication with the experimental crew. Non-normal and emergency communications was provided by a staff member. Aircraft were landing on Runways 13L and 22L and departing Runway $13 \mathrm{R}$ at KJFK. This configuration is typically used at Kennedy Airport for arrival priority.

All communications except with the tower were conducted using a form of pilot controller datalink. All procedures were designed to replicate future complex national airspace procedures with maximum traffic densities in accordance with goals for assessing usability in complex situations.

\section{RESULTS}

TPS was evaluated with typical ATC speed and altitude restrictions in context to the overall flight goals, with and without non-normal operations. The results presented represent subjective measurement of workload, acceptability and usability, and use in support of decision making. Subjective results are presented using an interval plot showing a 95\% confidence interval for the mean of each pilot role (pilot flying, pilot monitoring). Objective measurements were evaluated over one part of the arrival phase only - comparing fuel savings with and without the TPS indicators in the presence of an ATC speed reduction instruction.

\section{A. Workload}

Workload was measured using an industry standard multiindex measurement developed by NASA [14] and designated as task load index, or TLX. Fig. 3 shows the overall workload score for both pilot flying and pilot monitoring. The rating data indicate moderate levels of workload, even in the presence of a complex arrival in high volume airspace. Although not detailed 
in graphical form, there was no additional workload evident when using the TPS indicators as compared to the baseline workload.

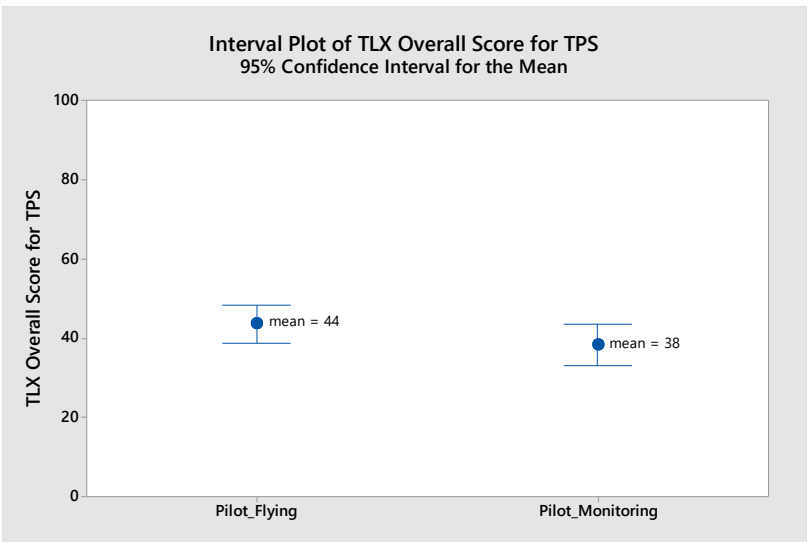

Fig. 3. Workload Scores

\section{B. Acceptability}

Acceptability was measured using a standard Likert-type scale from 1 to 7 , where 1 was very unacceptable, 4 was average, and 7 was very acceptable. Fig. 4 shows the acceptability rating data reported while using the TPS. TPS scored just under very acceptable for both pilot flying and pilot monitoring. The high acceptability rating was reinforced by pilot comments. For example, "I really look forward to this technology coming on line. Many pilots transitioning to advanced technology airplanes lack a fundamental understanding of the complex nature of a 3D environment. This helps shore up that lack of foundation." and "This is a significant improvement to the current symbology and information provided. I would use this capability every day and every flight to provide a more efficient and comfortable flight." These results align with findings from prior studies, albeit in lower fidelity simulations [3][8].

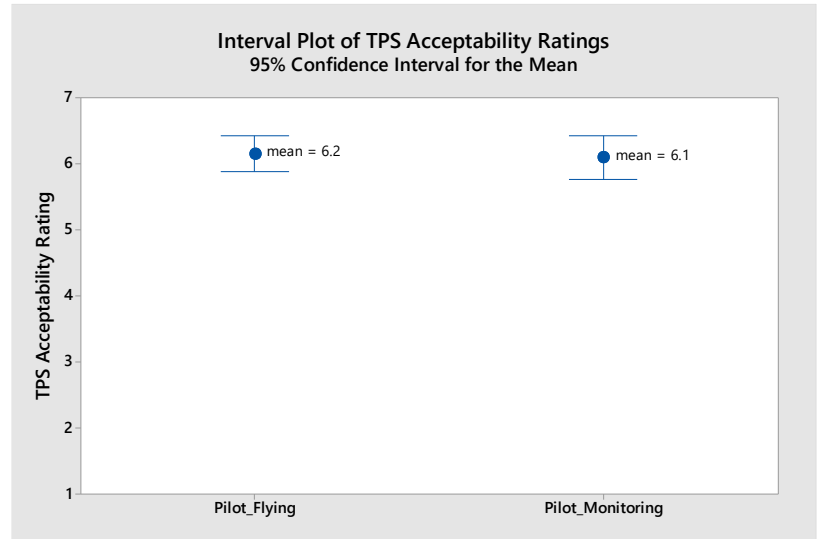

Fig. 4. Acceptability Ratings

Although the majority of pilot comments were positive, there were some negative comments. Some had to do with training and are often heard in similar studies where proper training is difficult to achieve in the limited time available. Most pilots concentrated on the vertical aspects; some pilots thought the information on the lateral portion of the navigation display was not useful. This is not surprising since the focus of this study was on an arrival segment. The following are the entirety of the negative comments from all twenty pilots: "TPS was more useful after experience. Too much information at first and not intuitive.", "No reason for both ALT CAP and ALT HOLD.", and "I was very satisfied using the TPS function on the VSD. I don't remember using it on the ND. I felt the ND was cluttered."

\section{Usability}

The System Usability Scale (SUS) [15] was used to gauge how pilots perceived usability of the TPS and associated indicators. SUS scores were calculated and range from 0 to 100 , but these were not percentile ranks. However, SUS scores can be associated with specific letter grades and adjective ratings [16]. As described in [16], an SUS score between 63 and 80.3 is considered a "good" design and a score above 80.3 is considered an "excellent" design.

Based on this criteria, TPS was rated an excellent design by both pilot flying and pilot monitoring, see Fig. 5. This result was supported by pilot comments such as "A picture is worth a thousand words and what the TPS shows is great. I know instantly if I'll make a crossing restriction and what mode will happen when", "TPS was very helpful, particularly on nonprecision approaches. Was very easy to understand information and provided a much more accurate representation verses standard flight path indicator", and "Loved this option. Its great big/small picture situation awareness tool. Helps conserve fuel and help crews meet complex restrictions because of better planning. Good that it tells you what to expect on FMA; help monitor changes or inaccuracies."

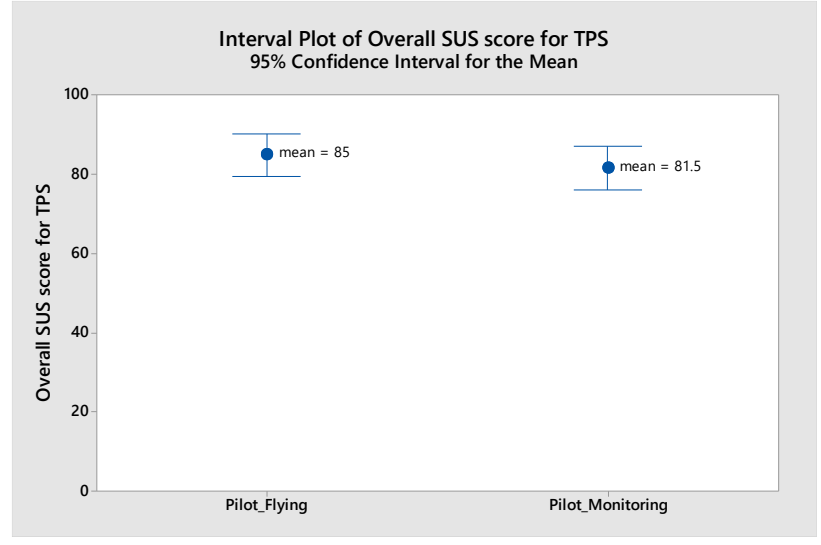

Fig. 5. System Usability Scores

\section{Decision Making}

Post-test questionnaires probed whether TPS was useful in support of decision making, in particular, for factors deemed important to energy management. From a list of 10 factors, pilots were asked to rate the top three factors for decisionmaking after each experimental run. From these questionnaires, the top factors cited for TPS are summarized in Fig. 6. (The summary graphic shows the most-often selected factors over all of the flights and how many times each of these factors was rated first, second, and/or third.) Over $80 \%$ of the pilot responses corresponded with the TPS goal of supporting better awareness 


\section{Top Decision-Making Factors}

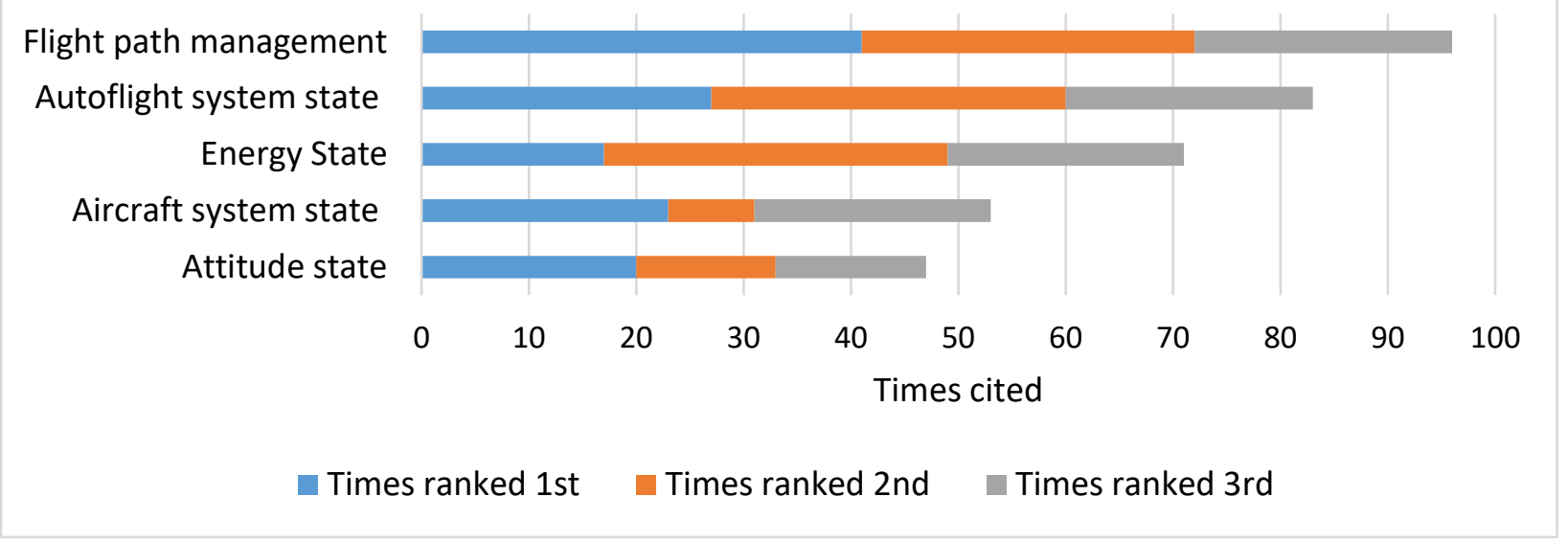

Fig. 6. Decision Making Factors

of the flight management system and auto-flight system states. Pilot comments reinforced this response. "The TPS allowed me to minimize the use of speedbrakes and encouraged me to continue utilizing VNAV instead of FLT LEVEL CHANGE $==>$ cost savings (fuel). It's an effective tool in efficiency allows us to notify ATC whether or not we can meet crossing restrictions", "I like using this for energy management. Often pilots throw speed brakes dive down. This will help fuel efficiency and passenger comfort as well as complex restriction compliance", and "I was able to see a vertical profile and its change in response to my input. Ability to see current path vs required path helped me manage my speed and energy state."

\section{E. Fuel Savings}

Although the study was not designed to test fuel savings, many of the pilot comments mentioned potential fuel savings as a benefit of TPS. Without TPS and presented with the upcoming altitude restriction at CAMRN and the aircraft trending high on the path while decelerating to meet the ATC speed restriction, all pilots extended speed brakes until the aircraft was back on path. With TPS, pilots could determine they were going to make both the altitude and speed restriction at CAMRN without using additional drag devices. Although this was only a very short segment and represented eight thousand feet of altitude loss, the difference between using speedbrakes to get back on path and allowing the automation to make the altitude restriction with no intervention amounted to 500 pounds of fuel saved.

Other pilot comments supporting this savings were "This was great for planning purposes and should save greatly on fuel costs. Not only does it help with planned top of descent, but long-term planning for an approach." and "TPS is a great tool for crossing restrictions and airspeed changes. It would work best if set up before descent."

\section{F. Display Clutter, Distraction, and Awareness of Energy State}

Post-test questionnaires investigated if TPS was distracting or cluttered the VSD and ND. Likert-type scales were utilized where a rating of 1 was "very cluttered" and a rating of 7 was "not at all cluttered." Median values for clutter were reported as 6 for the Captain and 6.25 for the First Officer and the values showed that TPS provided no more clutter than the current VSD and ND. Distraction was probed in the same way (rating of 1 "very distracting" to 7 "not at all distracting") and median values were 6.5 for the Captain and 7 for the First Officer. Additionally, pilots were also asked to "rate the degree to which the (new) predictive trajectory displays (e.g., the green prediction lines and mode change indicators) on the ND and VSD changed your awareness of the aircraft's energy state compared to the baseline technology" where a rating of 1 was "decreased awareness a lot" and a rating of 7 was "increased awareness a lot". As seen in Fig. 7, First Officers rated the VSD augmented with TPS as substantially increasing their awareness of energy with a mean value of 6.8. The tight spread of values, especially the First Officers, shows the value of the TPS on the VSD for energy awareness.

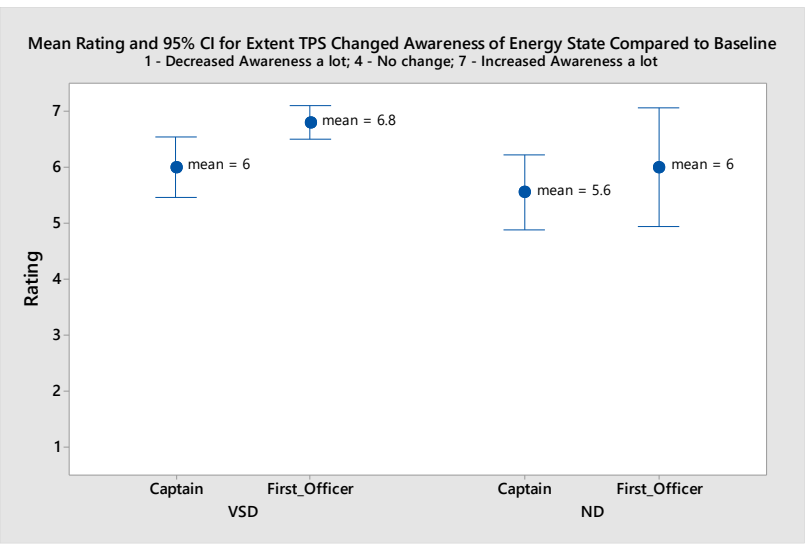

Fig. 7. Awareness of Energy State 


\section{CONCLUSION AND FUTURE RESEARCH}

Based on the pilot comments and the system usability measures, the automation state and trajectory prediction system was deemed useful in descent planning and execution, and was highly recommended for incorporation into aircraft systems. Although evaluated only for a short segment of the arrival, TPS showed the potential for fuel savings for even simple and routine ATC commands that are frequently issued daily in the national airspace system. While many pilots quickly forgot about the system and reverted to using drag devices when the workload was increased, further training and daily use could quickly lead to increased usage.

Pilot comments support some other additional design changes. The pilots felt that the textual information should be removed from the lateral portion of the navigation display and only the predicted path element should be retained. Consideration should be given to extending the predictor beyond five minutes to the next constrained waypoint during significant changing conditions. This feature would allow pilots to quickly evaluate if the future prediction will come close to meeting the constraints. Pilots should be trained in how speed changes affect path descents and they should be encouraged to resist initial use of speed brakes when in a deceleration phase. TPS shows how long the deceleration phase lasts so even using pictorials generated by TPS during prior flights or simulations could aid in training and encourage better planning.

Further study is needed to explore more aspects of the TPS for use in daily operational such as for climb and enroute phases of flight. If the addition of TPS to the VSD is considered across a fleet, a business as well as a safety case may be made, even for retrofit. Fuel savings result when pilots are not using drag devices and allowing the FMS to stay as high on the path as possible for the greatest amount of time even in the presence of ATC interventions. Fuel savings may be validated by using a few days of actual aircraft arrivals with actual ATC clearances. This validation could be done without a human-in-the-loop study using simulator models for performance, with and without the use of speed brakes for the arrivals.

Finally, arrival procedures are becoming more and more complex. Increasing traffic densities, interaction with adjacent airports, and noise complaints are just some of the issues driving this complexity. In the vertical dimension, this complexity results in multiple speed and altitude constraints, often requiring adherence to exact altitudes and airspeeds. These procedures do not always work in all environmental or traffic conditions, and ATC must often issue clearances that change the current plan. Without visibility into the required vertical profile and the automation's ability to perform to the plan, aircraft are being placed in a difficult situation with increased risk of loss of separation or even an accident. The VSD and TPS may be powerful tools as indicated by pilot feedback and other data reported here.

Reduced costs and increased safety support both forward-fit and retrofit of both the VSD and TPS capabilities.

\section{ACKNOWLEDGMENT}

This work was sponsored by NASA's Airspace Operations and Safety Program, System-Wide Safety project, led by John Koelling, Project Manager. This work is also covered in part under an Inter-Agency Agreement with the Federal Aviation Administration. Special thanks to Sabreena Azam, Ashley Awward, and John Stuber of the FAA for their support and guidance along the way.

\section{REFERENCES}

[1] United States Air Force Chief Scientist, "Report on Technology Horizons: A Vision for Air Force Science and Technology during 2010-2030" AF/ST-TR-10-01, Vol. 1, 15 May 2010.

[2] K. Abbott, "Human Factors Engineering and Flight Deck Design", The Avionics Handbook, C. Spitzer, Editor, Boca Raton: CRC Press LLC, 2001, pp.9.1-9.15.

[3] K. Shish, J. Kaneshige, D. Acosta, S. Shuet, T. Lombaerts, 1. Martin, m. Panda. "Trajectory Prediction and Alerting for Aircraft Mode and Energy State Awareness", Intelligent Interactions between Humans and Machines Conference, AIAA 2015 InfoTech.

[4] National Research Council, "Autonomy Research for Civil Aviation: Toward a New Era of Flight," 2014.

[5] Comercial Aviation Safety Team, "Safety Enhancement SE-208: Airplane State Awaress" www.skybrary.com.

[6] S. Young, "Flight Simulation Study of Airplane State Awareness and Prediction Technologies," Proceedings of the $35^{\text {th }}$ IEEE/AIAA Digital Avionics Systems Conference (DASC), Sacramento, CA, September 2016.

[7] T. Evans, L. Kreamer, T. Etherington, T. Daniels, S. Young, Y. Espada, J. Barnes, "Usability Evaluation of Indicators of Energy Related Problems in Commercial Flight Decks", 2019 IEEE/AIAA 38th Digital Avionics Systems Conference (DASC), San Diego, CA, USA, Sept 2019.

[8] J. Kaneshige, J. Benavides, S. Sharma, L. Martin, R. Panda, M. Steglinski, "Implementation of a Trajectory Prediction Function for Trajectory Based Operations," AIAA Aviation Atmospheric Flight Mechanics Conference, No. AIAA 2014-2198, August 2014.

[9] D. Carbaugh, S. Chen, A. Jacobsen, R. Myers, J. Wiedemann, "Vertical Situation Display" AERO 20, Boeing Magazine.

[10] T. Prevot, E. Palmer, "Staying Ahead of the Automation :A Vertical Situatoin Display Can Help", 2000-01-5614, Society of Automotive Engineers.

[11] Vakil, S. S., Midkiff, A. H. and Hansmann, R. J. (1996) Development and Evaluation of an Electronic Vertical Situation Display. NASA GRANT NAG1-1581

[12] T. Prevot, "A Display for Managing the Vertical Flight Path - an Appropriate Task with Inappropriate Feedback -“, International Conference on Human-Computer Interactions in Aeronautics, Montreal Canada, 1998.

[13] R. Neville and M. Day, "Innovative 787 Flight Deck Designed for Effeciency, Confort, and Comminality", Boeing Aeromagazine, March 2012 M. Park and J. Ostrower, "Air France Superjumbo Engine Failure Forces Emergency Landing in Canada", CNN, October 2017.

[14] Hart, S.G. and Staveland, L.E., "Development of NASA-TLX (Task Load Index): Results of Empirical and Theoretical Research.” In P.A. Hancock \& N. Meshkati (Eds.), Human Mental Workload. Amsterdam: NorthHolland, 1988, pp. 139-183.

[15] "System Usability Scale (SUS)," https://www.usability.gov/how-to-andtools/methods/system-usability-scale.html.

[16] J. Sauro, A Practical Guide to the System Usability Scale, Denver: Measuring Usability, 2011 